\title{
Modelling-Guided Design of Paper Microfluidic Networks - A Case Study of Sequential Fluid Delivery
}

\author{
Dharitri Rath ${ }^{1 \dagger}$, Bhushan J. Toley ${ }^{1 *}$ \\ ${ }^{1}$ Department of Chemical Engineering \\ Indian Institute of Science \\ Bengaluru, Karnataka 560012 \\ India
}

Keywords: Paper-based microfluidics, MicroPADs, Two-dimensional paper networks, Point-ofcare diagnostics, Richards equation, Partial saturation, Malaria diagnostics

\author{
* Correspondence to: \\ Bhushan J. Toley \\ Department of Chemical Engineering \\ Indian Institute of Science \\ C V Raman Avenue \\ Bengaluru, Karnataka 560012 \\ Phone: +91-80-2293-3114 \\ E-mail: bhushan@iisc.ac.in
}

\footnotetext{
${ }^{\dagger}$ Current address of Dharitri Rath is:

Department of Chemical Engineering

Indian Institute of Technology Jammu

Jagti, NH-44, PO Nagrota

Jammu - $181221 \mathrm{~J} \& \mathrm{~K}$, India
} 


\begin{abstract}
Paper-based microfluidic devices are popular for their ability to automate multi-step assays for chemical or biological sensing at a low cost, but the design of paper microfluidic networks has largely relied on experimental trial and error. A few mathematical models of flow through paper microfluidic devices have been developed and have succeeded in explaining experimental flow behaviour. However, the reverse engineering problem of designing complex paper networks guided by appropriate mathematical models is largely unsolved. In this article, we demonstrate that a two-dimensional paper network (2DPN) designed to sequentially deliver three fluids to a test zone on the device can be computationally designed and experimentally implemented without trial and error. This was accomplished by three new developments in modelling flow through paper networks: i) coupling of the Richards equation of flow through porous media to the species transport equation, ii) modelling flow through assemblies of multiple paper materials (test membrane and wicking pad), and iii) incorporating limited-volume fluid sources. We demonstrate the application of this model in the optimal design of a paper-based signal-enhanced immunoassay for a malaria protein, PfHRP2. This work lays the foundation for the development of a computational design toolbox to aid in the design of paper microfluidic networks.
\end{abstract}




\section{Introduction}

Paper microfluidic devices are well-suited to the development of point-of-care diagnostics because of their ability to automate multi-step assays at a low cost ${ }^{1}$. In order to conduct complex multi-step chemical assays, innovative paper networks have been designed to program the movement of fluids ${ }^{2}$. One of the earliest and most popular devices for programming fluid delivery in paper is the multi-legged two-dimensional paper network (2DPN), originally described by the Yager, Lutz, and $\mathrm{Fu}$ research groups ${ }^{3,4}$. These 2DPNs are designed to have multiple inlets per outlet and are used to sequentially deliver multiple fluids over a test zone automatically, without manual intervention. In a classic demonstration of a $2 \mathrm{DPN}$, the arrival time of multiple reagents was

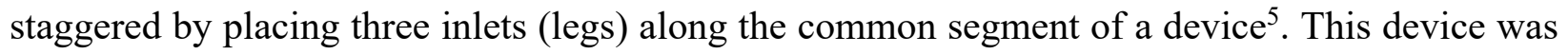
used for performing a signal-enhanced immunoassay for the detection of a malarial biomarker ${ }^{5}$. Subsequently Lutz et al. ${ }^{6}$ designed a 2DPN with legs of different lengths inserted into a shared buffer well; the legs disconnected from the source well in a timed sequence as the level of buffer in the well dropped, accomplishing sequential delivery of fluids to a test zone. A three-legged 2DPN was later used to develop an autonomous device for swab-to-result diagnosis of influenza using an immunoassay coupled with enzymatic signal-amplification ${ }^{7}$.

In parallel to use of new geometries, several efforts have been made to model wicking flow in paper microfluidics. The Washburn equation and Darcy's law are the two most commonly used methods $^{8-10}$. Dharmaraja et al. ${ }^{11}$ presented a framework for modelling wicking flow in paper networks using an electrical circuit analogy. An electrical circuit analogy was also used to model wicking through paper microfluidic shunts ${ }^{12}$. Elizalde et al. developed a model for imbibition through homogeneous porous substrates of arbitrary cross-sectional shape ${ }^{13}$. Future models 
incorporated the effects of partial saturation in paper caused by heterogeneous pore size distributions. Perez-Cruz et al. used the Richards equation, which solves for saturation (fraction of fluid-filled pores) as a function of space and time ${ }^{14}$. Tirapu-Azpiroz et al. ${ }^{15}$ demonstrated the use of the Richards equation to model flow in a three-legged 2DPN geometry. Cummins et al. developed a model to account for partial saturation by approximating paper by a collection of parallel capillaries of varying diameters stacked together ${ }^{16}$. Mendez et al. and Schaumburg et al. modelled wicking flow through paper by solving the Darcy's law using the moving boundary condition $^{10,17}$.

Despite the development of several mathematical models for flow through paper, design of multidimensional paper networks has largely relied on trial and error. Most modelling efforts till date have focused on developing models that can explain experimental data obtained from prefabricated devices. However, the inverse problem of designing a paper network that can accomplish a programmed set of fluidic operations guided by modelling has not been solved. This has primarily been the case because of the lack of appropriate models of imbibition through arbitrary shapes of 2DPNs. The use of the Richards equation to model flow in multidimensional paper networks represented a significant step forward in this direction ${ }^{14}$. However, in order to use the Richards equation, the capillary pressure and permeability of a paper material as a function of saturation must be known. Recently, simple experimental methods to measure capillary pressurepermeability-saturation relations for paper materials using minimal instrumentation were described by Buser ${ }^{18}$ and our group ${ }^{19}$. A combination of these experimental methods with Richards equation modelling overcomes the gap that has existed in this area for a long time and enables 
accurate modelling of imbibition through arbitrary shapes of paper networks fabricated from commercially available paper materials.

In the current work, we demonstrate the application of Richards equation in solving the inverse problem of designing paper networks to accomplish a desired set of timed fluidic operations. As a test case, we consider a 2DPN containing three inlets and one outlet intended for sequential delivery of three different fluids over a test zone. In order to accomplish this, three important advances were made in the field of modelling flow through paper: i) the Richards equation was coupled to the species transport equation enabling tracking the movement of multiple reagents in 2DPNs, ii) wicking flow was modelled through an assembly of two different porous materials representing the test membrane and the wicking pad, and iii) limited-volume fluid source wells that stopped flow after passage of a certain volume were incorporated at each of the three inlets. The model was used to solve two types of problems: a) for a given device geometry, to calculate the volumes of fluids to be delivered through the inlets for successful sequential delivery, and $b$ ) for a desired set of fluid volumes, to calculate device dimensions that would enable successful sequential delivery. For both cases, the predictions of the model were confirmed experimentally. We also demonstrate the application of these computational tools in designing a paper-based signal-enhanced immunoassay for the detection of a malarial protein, eliminating trial and error in the process of design. The approach developed here is general and can be used for the design of any multidimensional paper network intended to perform any set of fluidic operations. These methods bridge the gap between mathematical modelling and design in the field of paper microfluidics. 


\section{Experimental section}

\section{Materials and fabrication}

GE Whatman nitrocellulose membranes (NC FF120HP), cellulose pads (CF1, CF3) and glass fiber pads (GF/DVA) were procured from Wipro GE Healthcare Pvt. Ltd. (Bengaluru, India). Millipore cellulose pads CFSP (new code C083) were acquired from Sigma-Aldrich. All paper strips were laminated with pressure-sensitive adhesive (PSA; 3M $\mathrm{M}^{\mathrm{TM}}$ 9731). Transparent acrylic sheets used for preparing source liquid wells were acquired locally. All paper designs were drawn using AutoCAD (Autodesk, San Rafael, CA) and cut using a 50W CO2 laser cutter (VLS 3.60; Universal Laser Systems, Scottsdale, AZ).

\section{Diffusivity measurements}

Red, yellow, and orange food coloring dyes were acquired locally and their diffusivity in watersoaked nitrocellulose was measured using methods described in Supporting Information (SI) S1.

\section{Modelling flow through an assembly of test membrane and wicking pad}

The Richards equation was used to model flow through assemblies of nitrocellulose test strips connected to cellulose wicking pads at one end. In order to accomplish this, Van Genuchten parameters that relate capillary pressure and permeability to saturation must be known for all materials in the assembly. While they have been reported for NC FF120 by our group previously ${ }^{19}$, these parameters for the three wicking pad materials used here (CFSP, CF3 and CF4) were unknown. Therefore, the parameters for these materials were first determined and validated using a series of experimental and mathematical modelling methods previously published by our group ${ }^{19}$ (SI S2). After obtaining these parameters, flow was modeled through assemblies of NCFF120 
strips $(0.8 \mathrm{~cm} \times 12 \mathrm{~cm})$ connected to rectangular wicking pads $(4 \mathrm{~cm} \times 5 \mathrm{~cm})$ of CFSP, CF3 or CF4 using the Richards equation. The functional form of the Richards equation used throughout this work is as follows:

$$
\frac{\partial \theta}{\partial t}=\nabla \cdot\left(\frac{\kappa(\theta)}{\mu} \nabla(\psi(\theta))\right)
$$

where $\theta$ is saturation, defined as the fraction of the total volume of the strip occupied by fluid $(0 \leq$ $\theta \leq \theta_{S}$, where $\theta_{S}$ is the porosity). The value of saturation is normalized by defining: $S e=(\theta-$ $\left.\theta_{r}\right) /\left(\theta_{s}-\theta_{r}\right)$, where $\theta_{r}$ is the residual moisture content in the strips (assumed to be zero), therefore $S e=\theta / \theta_{s}$. Further, $\psi$ and $\kappa$ are capillary pressure and permeability of the material, respectively, and $\mu$ is the viscosity of the fluid. The equation was solved for $\theta$ over space and time using the "Richards equation interface" in the "subsurface flow module" of COMSOL v5.4. To validate these models, the corresponding devices were fabricated and flow through them was visualized. One complication in measuring flow rates through such an assembly is that once the fluid front moves from the test membrane into the wicking pad, it is no longer visible, and it becomes impossible to measure flow rates. To circumvent this issue, an electrochemical band marking setup that generated pink bands in a yellow fluid was used, similar to the one described by Kauffman et al. ${ }^{20}$ (see details in SI S3).

\section{Incorporating limited-volume source wells}

Limited-volume source wells were incorporated in the mathematical model using the following method. The volume of fluid entering a channel was calculated as:

$$
V=\int_{0}^{t}(v(t) \cdot A) d t
$$


where $\mathrm{v}(t)$ is the velocity of fluid averaged over the cross-section area of the paper membrane as a function of time, as estimated by solution of the Richards equation, and $A$ is the cross-sectional area of the inlet (considering thickness of the membrane as $100 \mu \mathrm{m}$ ). A logical condition was imposed on this boundary to cut flow off after the entering volume exceeded a predefined volume, say $60 \mu 1$. This was accomplished by reducing the permeability of a section of the device near the inlet to a very low value of $10^{-12} \mathrm{~m}^{2}$. This approach was experimentally validated on a single straight NCFF120 channel into which $60 \mu 1$ fluid, which was less than the fluidic capacity of the channel, was introduced from one end. The strip was placed in a humidity chamber and flow was recorded using a webcam. Note that an alternative to setting the permeability to a low value to stop flow is to apply a no-flux condition, which has been utilized to model the imbibition of a sessile droplet into paper ${ }^{21}$.

\section{Modelling species transport in paper membranes}

In order to model species transport in paper membranes, the Richards equation was coupled to the species transport equation:

$\frac{\partial\left(\theta C_{i}\right)}{\partial t}=\nabla \cdot\left(D_{e f f, i} \nabla C_{i}\right)-v \cdot \nabla C_{i}+R_{i}$

where $\theta$ is saturation, $v$ is the convective velocity, $C_{i}$ is the concentration, $D_{e f f ; i}$ is the effective diffusivity, and $R_{i}$ is the rate of reaction of the $i^{\text {th }}$ species. $R_{i}$ was set to zero for all species for all models in this work. The concentration term on the left side of the equation is multiplied by $\theta$ to account for partial saturation in porous media. The derivation of equation 3 from first principles is provided in SI S4. The convective velocity, $v$, is calculated and stored as a variable by the Richards equation interface in COMSOL, using the following expression:

$v=\left(\frac{\kappa(\theta)}{\mu} \nabla(\psi(\theta))\right)$ 
In order to obtain the spatiotemporal variation of saturation and concentrations of dissolved colored dyes, the coupled equations were solved in COMSOL using 'Richards equation interface' and 'Transport of diluted species in porous media interface' using the feature called 'Partially saturated porous media'. The parameters required for simulation are mentioned in SI Table S2. In cases where transport of multiple species was modelled, a separate species transport equation was written for each species. Note that the coupling with Richards equation is one-way, i.e. the Richards equation may be solved without any input from the species transport equations, but the species transport equations require values of convective velocity from the Richards equation. The following boundary conditions were used for the transport equations: $C_{i}=1 \mathrm{~mol} / \mathrm{m}^{3}$ at the inlet, and an outflow boundary condition $\left(\vec{n} \cdot D_{\text {eff }} \nabla C_{i}=0\right)$ was set at the outlet.

Prior to modelling multiple dye solutions in 2DPNs, coupling of Richards equation with the species transport equation was experimentally validated using a straight NC FF120 channel (10 cm x 0.8 $\mathrm{cm}$ ), in which a single red-colored dye solution was introduced from one end. Flow was stalled at different pre-determined time points by rapidly drawing fluid out of the source-well using a Kimwipe. The strip was immediately cut into 1-cm sections using pre-marked graduations. Fluid from each 1-cm section was removed from the paper by centrifugation and weighed to measure the saturation in that section, similar to a method described previously by our group ${ }^{19}$. Additionally, concentration of the dye in the centrifuged fluid was measured by measuring the absorbance at $520 \mathrm{~nm}$ using a 96 well plate reader equipped with a $\mu$ DROP plate adapter (Multiskan Sky Microplate Spectrophotometer, Thermo Scientific). For quantification, a calibration curve for concentration vs absorbance was generated and linearity was ensured within the concerned concentration range (SI S5). A schematic of the procedure is presented in SI S6. 


\section{Transport and sequential delivery in 2DPNs}

The techniques developed above were then used to model flow in 2DPNs having three inlets, intended for sequential delivery of three fluids to a common test region. Each fluid was considered to be constituted of a single chemical species dissolved in water. Two types of inverse problems were solved - i) estimation of volumes for a fixed device geometry and ii) estimation of key geometric parameters for fixed volumes, to accomplish successful sequential delivery. In case i, volumes input into legs 1 and 2 were set as parameters and were varied in between 10-25 $\mu 1$; volume input into leg 3 was set to a large value of $70 \mu$ l. For case ii, the distances between legs 1 and 2, and between legs 2 and 3, were set as parameters and varied between $0.5-2.5 \mathrm{~cm}$. In order to simultaneously visualize the location of the three fluids in the results of the model, the following post-processing was conducted. Separate grayscale movies of the concentrations of individual species were generated in COMSOL. The three grayscale movies, corresponding to concentrations

of the three different species, were merged in ImageJ using the 'Merge color channels' option, and each source movie was assigned a unique color. This generated a colored movie showing the realtime location of all three fluids. After solving the models over a large set of parameter values, devices corresponding to a few key parameters were fabricated for model validation.

\section{Results and Discussion}

\section{Modelling flow through assemblies of test membranes and wicking pads}

To ensure rapid and continuous flow from a test membrane into a wicking pad, the choice of wicking pad material is important. Three different cellulosic wicking pad materials were considered - CFSP, CF3, and CF4. Capillary pressure vs saturation curves, permeability vs 
saturation curves, and Van-Genuchten parameters for the three materials are provided in SI S2. The parameters were first validated by comparing experimentally and computationally-obtained spatiotemporal saturation profiles in rectangular strips of these materials using methods developed by our group previously ${ }^{19}$; the profiles matched well for all three materials (Figure 1A-C).

Flow rates through assemblies of NCFF 120 attached to either of the three wicking pad membranes were then experimentally measured using the band-marking setup (SI Movies S1, S2, S3). From $t$ $=0$ to $\sim 170 \mathrm{~s}$, fluid fronts were in the NCFF120 membrane, over which time the velocities continuously decreased (Washburn behavior; Figure 1D). Subsequently, after entering the wicking pad, there was a sudden deviation from Washburn behavior. The rate of reduction in velocity generally decreased; this state may be referred to as a quasi-stationary state ${ }^{10}$ and CFSP produced the highest velocity in this state (Figure 1D). Because high flow rates are desired for rapid completion of multi-step assays, CFSP was chosen as the wicking pad material for all 2DPNs in this work. Flow through the above assemblies was then computationally modelled (Figure 1E). It is of note that the CFSP wicking pad is significantly thicker $(830 \mu \mathrm{m})$ than the NC flow strip (100 $\mu \mathrm{m})$ and acts as a close-to-infinite fluid sink. In order to avoid modelling a 3D domain, the experimentally used 3D wicking pad $(2 \mathrm{~cm} \times 4 \mathrm{~cm} \times 0.83 \mathrm{~mm})$ was approximated by a much longer 2D wicking pad $(2 \mathrm{~cm} \times 8 \mathrm{~cm})$ in the model to ensure a close-to-infinite fluid sink. Results of the model captured the following key features of experimental data: i) continuous reduction in velocity when fluid fronts are in NCFF120, ii) quasi stationary flow after fluid fronts enter wicking pads, and iii) maximum velocity in the quasi stationary state when CFSP is used as the wicking pad (Figure 1E). This is the first model of wicking flow through assemblies of different paper materials and marks an important advancement in the field. 

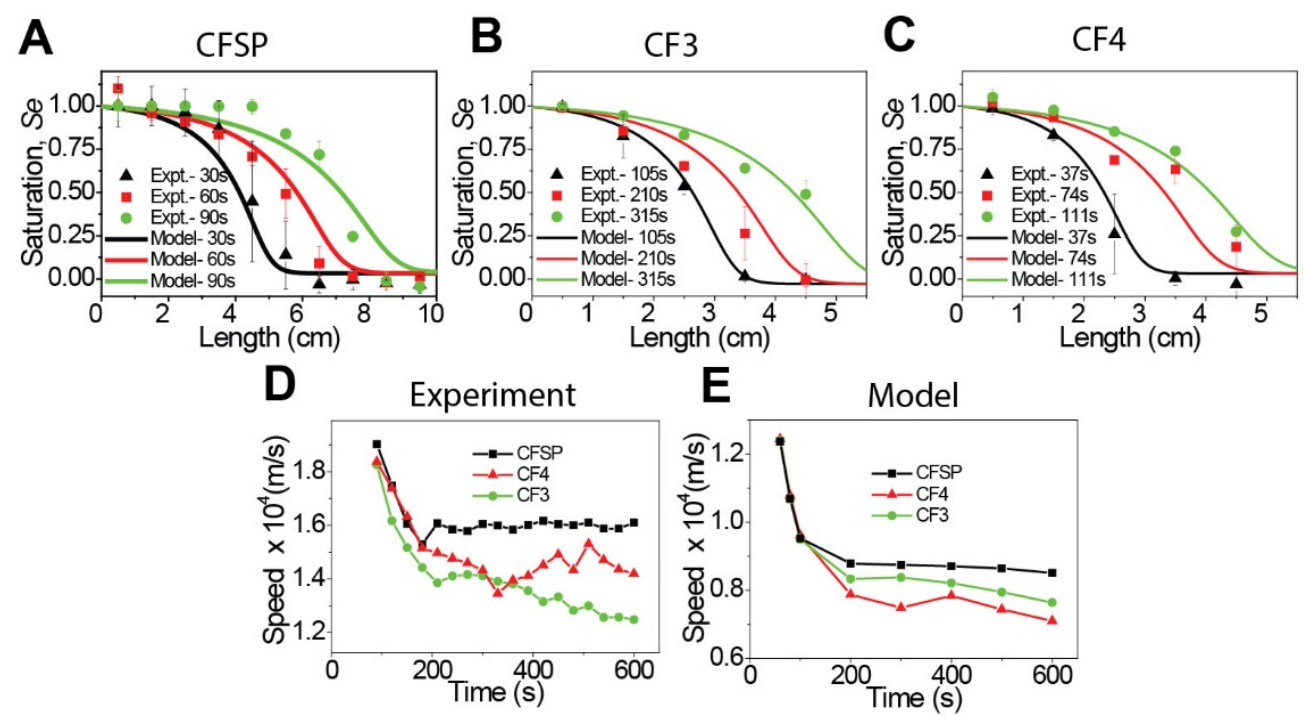

Figure 1: Characterization of wicking pads. A-C. Spatiotemporal variation of saturation in strips of (A) CFSP, (B) CF3 and (C) CF4 membranes. D-E. Experimental (D) and COMSOL modeling (E) results of the speeds of movement of the fluid front for an assembly of a test membrane (nitrocellulose NC FF120) attached to three wicking pad materials - CFSP, CF3 and $\mathrm{CF} 4$.

\section{Incorporating limited-volume source wells}

Till date, all models of flow in paper microfluidic devices have assumed infinite-volume fluid sources. However, incorporation of limited volume source wells is critical for modelling 2DPNs designed for sequential delivery of fixed fluid volumes to a test zone. Incorporation of a limitedvolume source well was first validated using flow in a straight channel. When a limited volume $(60 \mu 1)$ of fluid was introduced into an NCFF120 strip, it drained into the strip over 390 seconds, following which movement of fluid stopped (Figure 2A). Spatiotemporal saturation plots predicted by a model that uses a logical boundary condition to incorporate a limited volume fluid source are shown in Figure 2B. There was a strong match in experimental and simulated location of the fluid front as a function of time (Figure 2C), validating the approach. This is an important result because 
it enables modelling flow in paper microfluidic devices connected to source wells of predefined volumes.
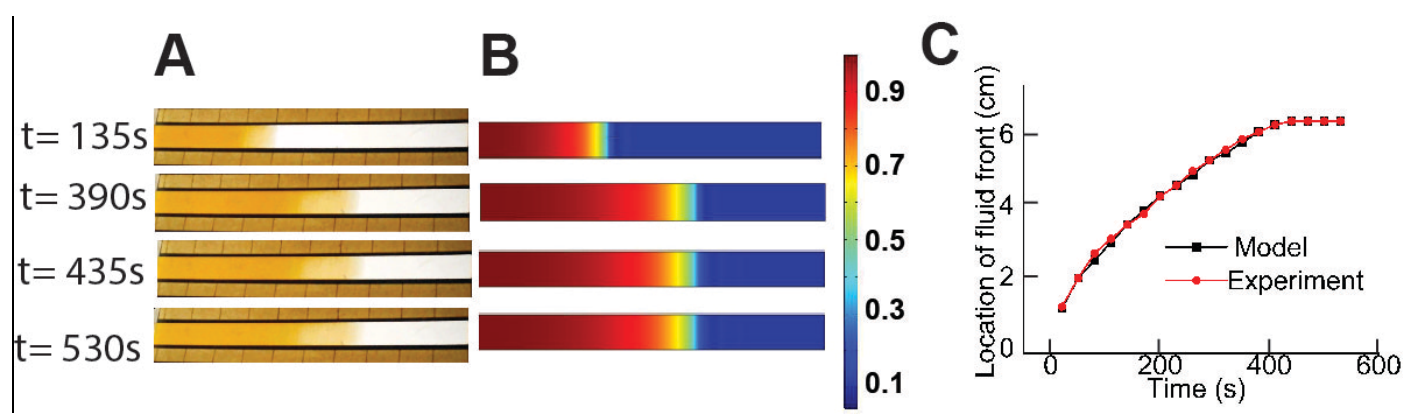

Figure 2: Modelling limited-volume fluid sources. A-B. Experimental (A) and modelled (B) time-lapse images of the advancing fluid front in an NC strip connected to a limited volume fluid reservoir. C. Comparison of the location of the advancing fluid front along the channel measured experimentally (red markers) and predicted using COMSOL simulations (black markers).

\section{Modelling species transport in paper networks}

To validate the coupling of species transport equation to the Richards equation, saturation and dye concentration were measured experimentally for two test membranes - NC FF120 and glass fiber GF/DVA. For the model, diffusion coefficients for red, yellow, and orange dyes were first measured experimentally and found to be $1.667 \pm 0.76 \times 10^{-10} \mathrm{~m}^{2} / \mathrm{s}, 3 \pm 0.5 \times 10^{-10} \mathrm{~m}^{2} / \mathrm{s}$, and $1.667 \pm 0.76 \times 10^{-10} \mathrm{~m}^{2} / \mathrm{s}$, respectively $(\mathrm{N}=3)$. Figure 3 shows experimental (markers) and mathematically modelled (lines) spatiotemporal profiles of saturation and dye concentration for NC FF120 (Figure 3A) and GF/DVA (Figure 3B). There was good agreement between experimental and simulated profiles and the model correctly captured the following important characteristics of experimental profiles: i) continuous and smooth reduction in saturation from a value of 1 to 0 with increasing length, and ii) a step change in the normalized concentration of dye from 1 to 0 coinciding with the location where saturation reached a value of 0 . The step change can be explained by the fact that there is no mechanism at play that would change the intrinsic dye concentration, i.e. moles of dye per volume of fluid. The normalized concentration of the dye in 
the fluid is independent of saturation and its value is binary -1 where fluid exists and 0 where no fluid exists. For experimental measurement, the fluid was first centrifuged out of the membrane, facilitating intrinsic concentration measurement. This is the first model that has accomplished simultaneous modelling of saturation and concentration and is an important tool for designing paper microfluidic networks containing multiple fluids, as subsequent sections will illustrate.

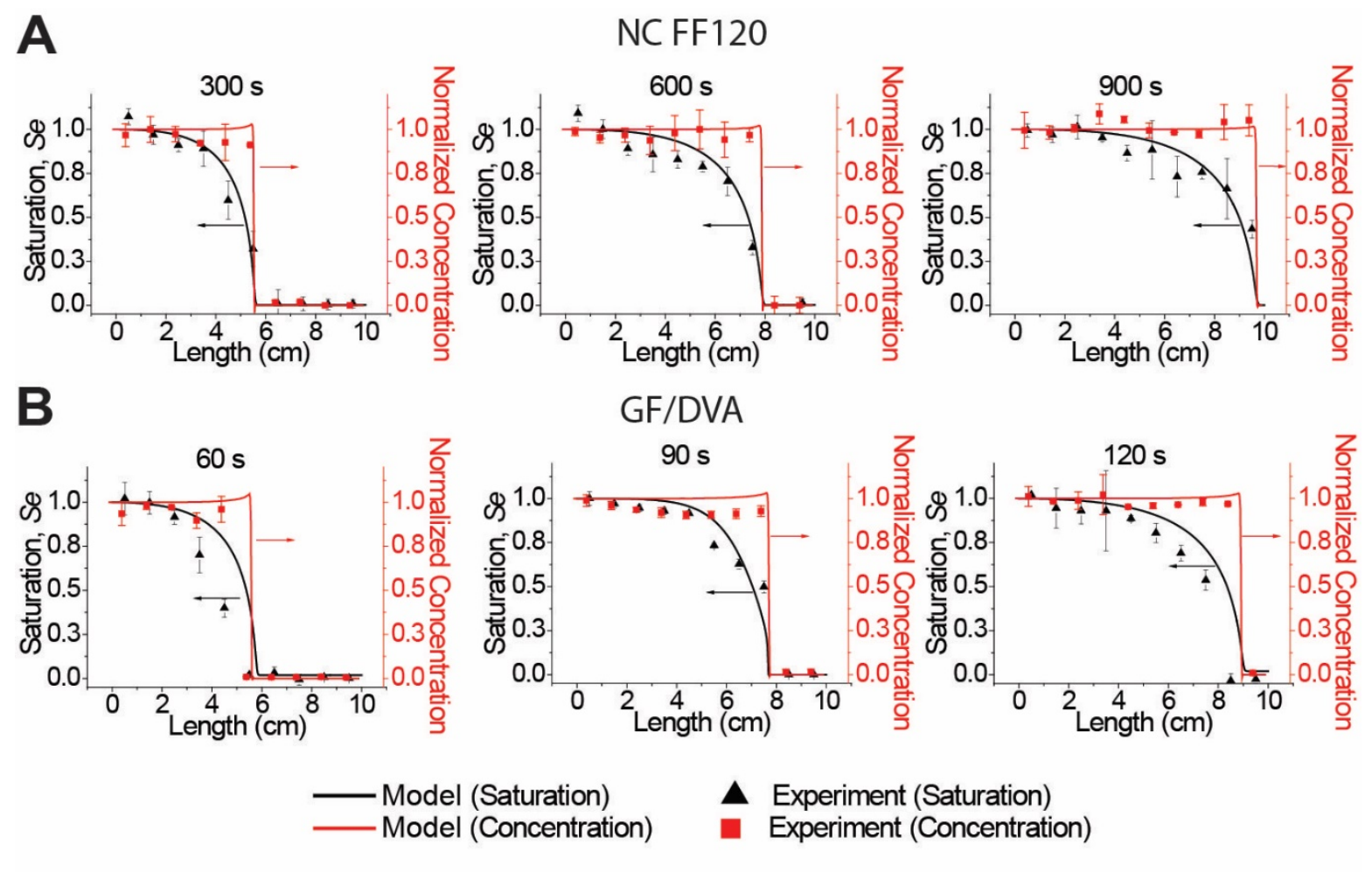

Figure 3: Validation of species transport modelling. A-B. Saturation (black; primary axis) and normalized concentration (red; secondary axis) along the lengths of NCFF120 (A) and GF/DVA (B) strips. In all panels, scatter plots show experimental data and line plots show modelling results. Experimental data was obtained by chopping the strip into 1-cm sections. Experimental data shown at $0.5 \mathrm{~cm}$ represents data from the $1^{\text {st }} 1-\mathrm{cm}$ section from the source, at $1.5 \mathrm{~cm}$ represents that from the $2^{\text {nd }}$ section, and so on. All error bars represent standard deviation $(\mathrm{N}=3)$.

\section{Modelling sequential delivery in 2DPNs}

After validation of the three new techniques, i.e. i) modelling flow through assemblies of two different paper materials, ii) modelling limited-volume fluid sources, and iii) coupling flow and 
species transport modelling, these techniques were utilized together to model sequential fluid delivery in a three-legged 2DPN. For this, consider the general three-legged 2DPN design in Figure 4A. For this work, the width of all flow channels in the design was set to $0.5 \mathrm{~cm}$ and design parameters were ' $a$ ' and ' $b$ ': the distances between legs 1 and 2 and between legs 2 and 3 , respectively; and $V_{1}, V_{2}, V_{3}$ : volumes of fluids introduced into reservoirs connected to legs 1,2 , and 3 , respectively.

For initial validation, we considered a 2DPN with $a=b=0.5 \mathrm{~cm}$ and two cases: I) $V_{l}=13, V_{2}=15$, and $V_{3}=70 \mu 1$ and II) $V_{1}=25, V_{2}=35$, and $V_{3}=70 \mu 1$. Experiments were performed for these two cases by introducing the corresponding fluid volumes into reservoirs connected to the legs of the devices and flow was recorded (Figure 4B, D). Simulations were then conducted to match these two cases (Figure 4C, E). Case I represents successful sequential delivery of the three fluids to the test line. After the three fluids were introduced into reservoirs, pink, yellow, and orange fluids existed at the test line at 120, 240, and 420s, respectively (Figure 4B). The corresponding modelling results match well (Figure 4C, SI Movie S4). Case II, on the other hand, represents a case of unsuccessful sequential delivery. Here, although pink fluid from leg 1 exists at the test line at $t=56 \mathrm{~s}$, there is coexistence of pink and yellow fluid at the test line at $t=240 \mathrm{~s}$ and of yellow and orange fluid at $t=540 \mathrm{~s}$ (Figure 4D). The modelling results match the experimental observation of coexistence of two fluids at the test line at $t=240$ and 540s (Figure 4E, SI Movie S5). Minor discrepancies may be observed in the exact location of fluid fronts between experimental and modelling results, possibly because of approximating the 3D wicking pad with a $2 \mathrm{D}$ pad. However, these minor discrepancies do not affect the practical application of the model in accurately predicting the success or failure of sequential delivery. 

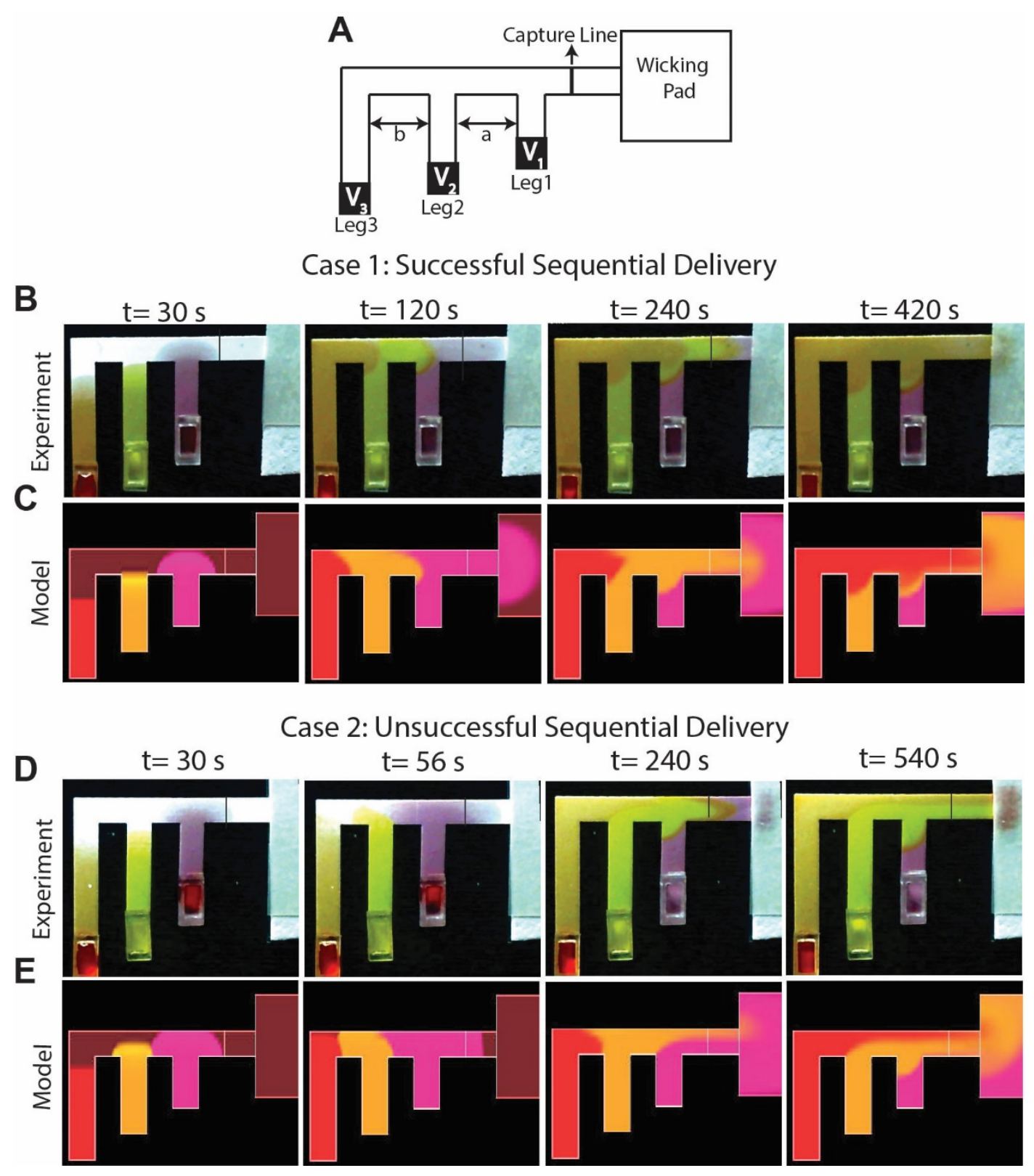

Figure 4: Sequential delivery of three fluids in a 2DPN. A. Schematic of a three-legged 2DPN showing design parameters $a$ and $b$ (distance between legs) and $V_{1}, V_{2}$, and $V_{3}$ (volumes of fluids delivered). B-C: Experimental (B) and modelled (C) results for a case of successful sequential delivery. Only one colored fluid exists at the capture line at any given time. D-E: Experimental (D) and modelled (E) results for a case of unsuccessful sequential delivery. Two fluids coexist at the capture line at 240 and 540 s.

After determining that the model could predict success or failure of sequential delivery, the first of the two design problems was solved, i.e. determination of optimum volumes for sequential 
delivery in a fixed network geometry. This was accomplished by solving the model over a range of parameter values for $V_{l}$ and $V_{2}$, keeping parameters $a$ and $b$ fixed to $0.5 \mathrm{~cm}$ and plotting average concentrations of the three dyes at the test line over time. In a hypothetical ideal case of sequential delivery, such a plot would appear as three adjacent square waves (Figure 5A). Modelling results from representative pairs of $V_{l}$ and $V_{2}$ are shown in Figure 5B, which reveals several modes of failure to deliver sequentially. When $V_{2}$ is too low $(10 \mu$ l), fluid from leg 2 fails to deliver (left column; Figure 5B); when it is too high $(20 \mu 1)$, fluid from leg 2 delivers to the test zone but continues to flow even after fluid from leg 3 reaches, leading to co-flow (right column; Figure 5B). Successful sequential delivery was possible for $V_{2}=15 \mu \mathrm{l}$ (middle column; Figure 5B), except in the last row, for which $V_{l}$ was too high $(20 \mu \mathrm{l})$ causing co-delivery of fluids from leg 1 and 2 . To make this analysis amenable to optimization algorithms, a single numerical parameter, $P$, representing the quality of sequential delivery was defined as $P=\left(C_{1, \text { avg }}\right.$ when $\left.\mathrm{C}_{1} \geq 0.1\right)+\left(C_{2, \text { avg }}\right.$ when $\left.\mathrm{C}_{2} \geq 0.1\right)+\left(\mathrm{C}_{3, \text { avg }}\right.$ when $\left.\mathrm{C}_{3} \geq 0.1\right) / 3$, where $C_{1}, C_{2}$ and $C_{3}$ are average concentrations of species 1,2 , and 3 over the test line and over time. For the hypothetical case of ideal delivery (Figure 5A), the value of $P$ is 1. $P$ values for a larger set of combinations of $V_{l}$ and $V_{2}$ are shown in Figure 5C. It was empirically determined that successful delivery occurred for $P$ values $>0.8$. Based on this, volumes for successful sequential delivery can be identified (green zone; Figure 5C) computationally. This was experimentally validated for one representative case of successful (SI Movie S6) and unsuccessful (SI Movie S7) sequential delivery. 

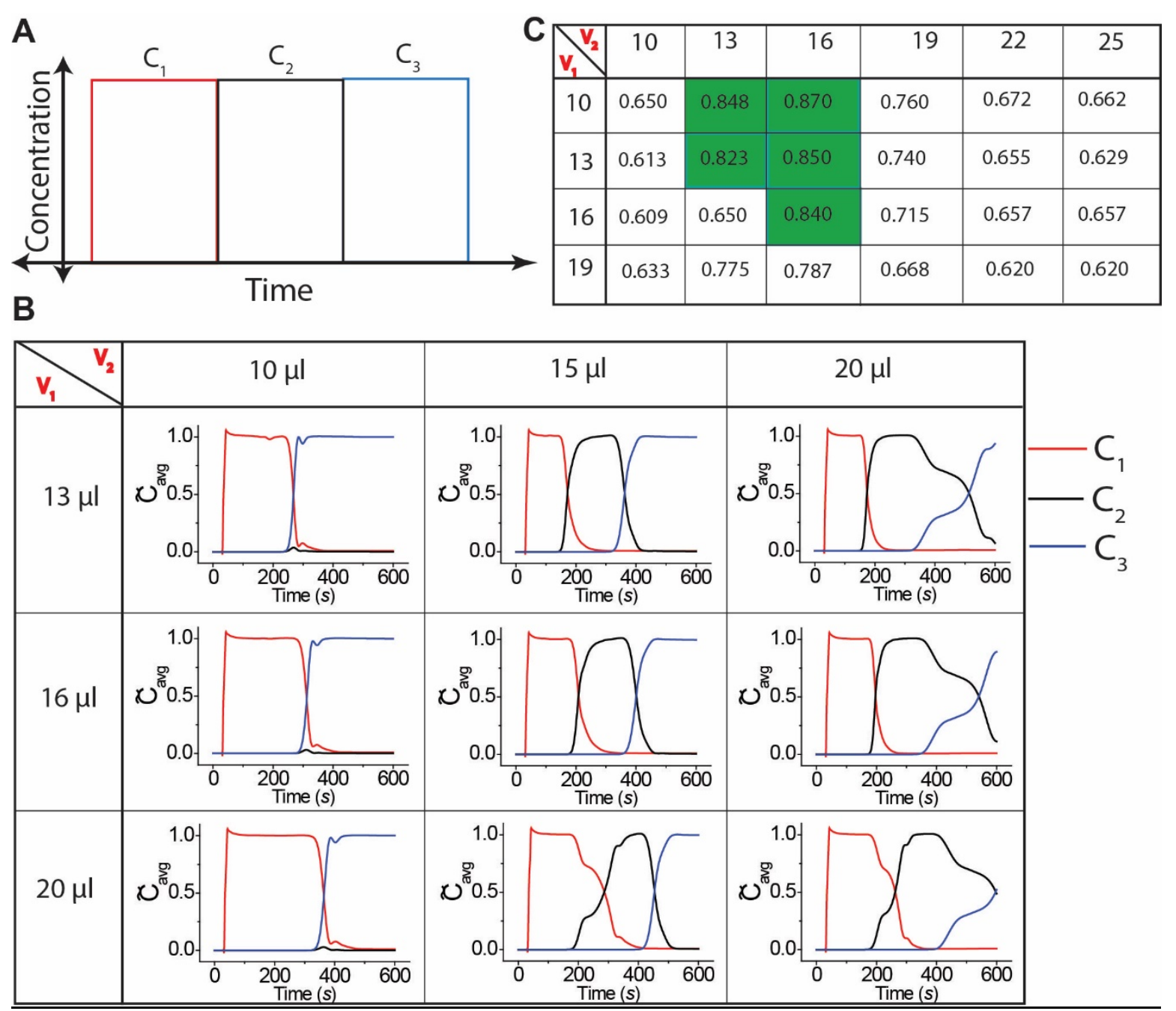

Figure 5: Results of modelling sequential delivery in a 2DPN. A. Schematic showing the temporal concentration profiles of the three reagents, $C_{1}, C_{2}$ and $C_{3}$, over the test line for a hypothetical case of ideal sequential delivery. B. Modelled temporal concentration profiles for representative combinations of $V_{1}$ and $V_{2}$. C. Parameter $P$ evaluated over a larger set of combinations of $V_{1}$ and $V_{2}$. A value of $P>0.8$ (green zone) indicates successful sequential delivery.

The second engineering problem of determining the geometry for the sequential delivery of fixed fluid volumes was solved next. This was accomplished by varying the values of parameters $a$ and $b$, keeping $V_{1}, V_{2}$, and $V_{3}$ fixed at 25, 25, and $120 \mu$, respectively. Simulated concentration-time profiles over the test line for various combinations of $a$ and $b$ and the corresponding $P$ values are shown in Figure 6A. Based on these results, successful sequential delivery $(P>0.8)$ was 
accomplished using five combinations of $a$ and $b$ (green zone, Figure 6A). A few representative cases were experimentally validated (SI Movies S8, S9, S10).

The application of effective sequential delivery is finally demonstrated in the context of a signalenhanced immunoassay for the detection of a malarial antigen Plasmodium falciparum HRP2 protein (PfHRP2) in 2DPNs. The detailed description of the assay is provided in the SI S7. Briefly, a sandwich immunoassay was performed wherein one antibody against PfHRP2 was conjugated to gold nanoparticles for visual detection and the other antibody was manually spotted as the capture antibody in the test zone. A $25 \mu 1$ sample containing a mixture of PfHRP2 antigen and the detection antibody conjugate was introduced into Leg 1. A wash solution (25 $\mu 1$ PBST) and gold signal enhancement reagents $(70 \mu \mathrm{l})$ were introduced into Legs 2 and 3, respectively. Two geometries, one representing unsuccessful $(a=b=0.5 \mathrm{~cm} ; P=0.683)$ and one representing successful $(a=b=1.5 \mathrm{~cm} ; P=0.860)$ delivery were chosen for conducting the assay. For the geometry that would cause unsuccessful sequential delivery, the test signal only developed in the bottom half of the test strip (Figure 6B (i)). For the other geometry enabling successful sequential delivery, the test signal developed uniformly over the full width of the test strip (Figure 6B (ii)). Partial signal generation in the test zone is a result of simultaneous delivery of antigen from Leg 1 and wash solution from Leg 2 to the test spot (as predicted by our model), only exposing the bottom half of the test spot to the antigen. Proper sequential delivery thus improves the performance of the multi-step immunoassay. 


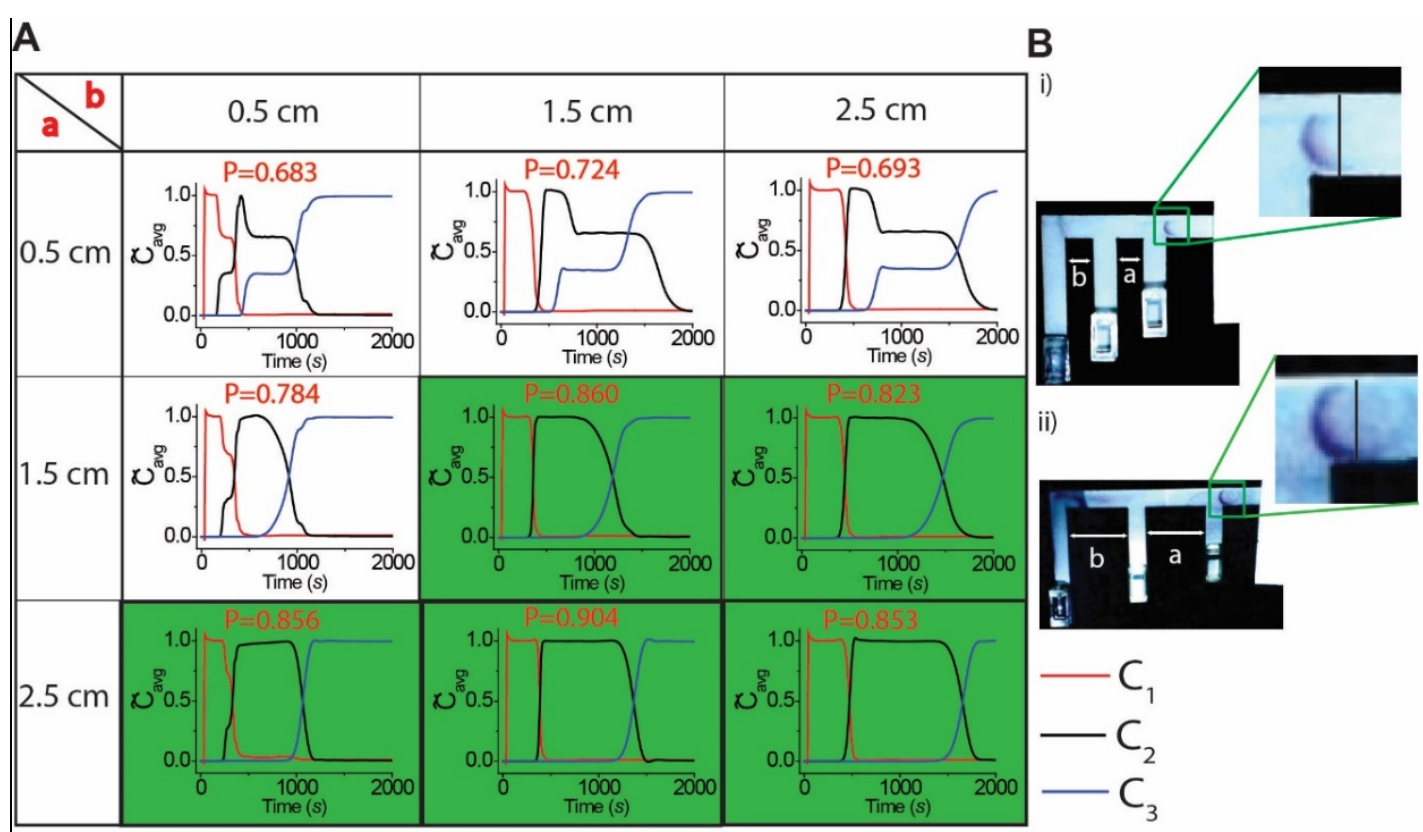

Figure 6: Modelling-guided design of a 2DPN to conduct a signal-enhanced immunoassay. A. Temporal concentration profiles of the three reagents, $\mathrm{C}_{1}, \mathrm{C}_{2}$ and $\mathrm{C}_{3}$, over the test line for $a$ few combinations of ' $a$ ' and ' $b$ '. B. Signal enhanced immunoassay performed on two 2DPN designs that are (i) unsuccessful and (ii) successful at accomplishing sequential delivery of reagents. The signal spot developed only over a fraction of the width of the test strip for unsuccessful sequential delivery (i) and over the entire width of the strip in the case of successful sequential delivery (ii).

While initial demonstrations in this work are restricted to fluids having properties close to water, the methods are extendible to fluids having different flow properties, e.g. body fluids like saliva, blood etc., which may be used as samples in paper microfluidic networks. In order to accomplish this, Van Genuchten parameters must first be obtained for each specific membrane-fluid pair because the surface tension of the fluid and contact angle with the membrane will affect these parameters. These parameters can be easily obtained using methods identical to those used in this work and will be the focus of future work in our lab. The viscosity of sample fluid may be different than that of water and that parameter can easily be changed as it appears explicitly in Equation 1 (Richards equation). Diffusivities would also change depending on the specific detection molecules and fluids used. However, transport phenomena are dominated by convection in these 
systems and diffusivities have an insignificant role to play (Peclet numbers are of the order of $10^{3}$; SI S8). Therefore, the values of diffusivities used in this study may directly be adopted for other systems. Finally, the extrapolation of these methods to 3D systems is straightforward using COMSOL as a modelling platform.

\section{Conclusion}

The methods described in this work enable computational design of multidimensional paper networks that can accomplish a programmed set of fluidic operations. Although demonstrated in the context of a three-legged 2DPN in this work, the applications of this method span the entire gamut of paper-based analytical devices. In combination with methods for modelling flow in paper analytical devices using Richards equation ${ }^{19}$, the new methods described in this work provide a complete toolbox to model the flow of multiple fluids through arbitrary shapes of paper networks. In addition to applications in design, these methods can also be used to enhance fundamental understanding of transport phenomena and reaction mechanisms in paper-based analytical devices in the context of partial saturation. Till date, all models of reactions within paper analytical devices have been oblivious to the phenomenon of partial saturation. The methods developed in this work could be used to overcome this gap in the field and will enable novel investigations into the effect of partial saturation on analytical chemistry performed in paper-based devices.

\section{Conflict of interest}

We have none to declare.

\section{Acknowledgement}


This work was supported by the Science and Engineering Research Board (SERB, India) in the form of an Extramural Research Grant (EMR/2016/006029) to BT and a National Postdoctoral Fellowship (PDF/2017/002181) to DR.

\section{References}

(1) Cate, D. M.; Adkins, J. A.; Mettakoonpitak, J.; Henry, C. S. Recent Developments in Paper-Based Microfluidic Devices. Anal. Chem. 2015, 87 (1), 19-41.

(2) Fu, E.; Downs, C. Progress in the Development and Integration of Fluid Flow Control Tools in Paper Microfluidics. Lab Chip 2017, 17 (4), 614-628.

(3) Fu, E.; Lutz, B.; Kauffman, P.; Yager, P. Controlled Reagent Transport in Disposable 2D Paper Networks. Lab Chip 2010, 918-920.

(4) Fu, E.; Kauffman, P.; Lutz, B.; Yager, P. Sensors and Actuators B : Chemical Signal Amplification in Two-Dimensional Paper Networks. Sensors Actuators B. Chem. 2010, $149(1), 325-328$.

(5) Fu, E.; Liang, T.; Spicar-Mihalic, P.; Houghtaling, J.; Ramachandran, S.; Yager, P. TwoDimensional Paper Network Format That Enables Simple Multistep Assays for Use in Low-Resource Settings in the Context of Malaria Antigen Detection. Anal. Chem. 2012, $84,4574-4579$.

(6) Lutz, B.; Liang, T.; Fu, E.; Ramachandran, S.; Kauffman, P.; Yager, P. Dissolvable Fluidic Time Delays for Programming Multi-Step Assays in Instrument-Free Paper Diagnostics. Lab Chip 2013, 13 (14), 2840-2847.

(7) Huang, S.; Abe, K.; Bennett, S.; Liang, T.; Ladd, P. D.; Yokobe, L.; Anderson, C. E.; Shah, K.; Bishop, J.; Purfield, M.; et al. Disposable Autonomous Device for Swab-to- 
Result Diagnosis of Influenza. Anal. Chem. 2017, 89 (11), 5776-5783.

(8) Fu, E.; Ramsey, S. A.; Kauffman, P.; Lutz, B.; Yager, P. Transport in Two-Dimensional Paper Networks. Microfluid. Nanofluidics 2011, 10 (1), 29-35.

(9) Toley, B. J.; Das, D.; Ganar, K. A.; Kaur, N.; Meena, M.; Rath, D.; Sathishkumar, N.; Soni, S. Multidimensional Paper Networks: A New Generation of Low-Cost Pump-Free Microfluidic Devices. J. Indian Inst. Sci. 2018, xxx, 1-34.

(10) Mendez, S.; Fenton, E. M.; Gallegos, G. R.; Petsev, D. N.; Sibbett, S. S.; Stone, H. a; Zhang, Y.; López, G. P. Imbibition in Porous Membranes of Complex Shape: QuasiStationary Flow in Thin Rectangular Segments. Langmuir 2010, 26 (2), 1380-1385.

(11) Dharmaraja, S.; Lafleur, L.; Byrnes, S.; Kauffman, P.; Buser, J.; Toley, B.; Fu, E.; Yager, P.; Lutz, B. Programming Paper Networks for Point of Care Diagnostics. In Proceedings of SPIE - The International Society for Optical Engineering; 2013; Vol. 8615.

(12) Toley, B. J.; McKenzie, B.; Liang, T.; Buser, J. R.; Yager, P.; Fu, E. Tunable-Delay Shunts for Paper Micro Fl Uidic Devices. Anal. Chem. 2013, 85 (23), 11545-11552.

(13) Elizalde, E.; Urteaga, R.; Berli, C. L. a. Rational Design of Capillary-Driven Flows for Paper-Based Microfluidics. Lab Chip 2015, 15, 2173-2180.

(14) Perez-Cruz, A.; Stiharu, I.; Dominguez-Gonzalez, A. Two-Dimensional Model of Imbibition into Paper-Based Networks Using Richards’ Equation. Microfluid. Nanofluidics 2017, 21 (5), 1-12.

(15) Tirapu-Azpiroz, J.; Silva, A. F.; Ferreira, M. E.; Candela, W. F. L.; Bryant, P. W.; Ohta, R. L.; Engel, M.; Steiner, M. B. Modeling Fluid Transport in Two-Dimensional Paper Networks. J. Micro/Nanolithography, MEMS, MOEMS 2018.

(16) Cummins, B. M.; Chinthapatla, R.; Ligler, F. S.; Walker, G. M. Time-Dependent Model 
for Fluid Flow in Porous Materials with Multiple Pore Sizes. Anal. Chem. 2017, 89 (8), 4377-4381.

(17) Schaumburg, F.; Kler, P. A.; Berli, C. L. A. Numerical Prototyping of Lateral Flow Biosensors. Sensors Actuators, B Chem. 2018, 259, 1099-1107.

(18) Buser, J. R. Heat, Fluid, and Sample Control in Point-of-Care Diagnostics, University of Washington, 2016.

(19) Rath, D.; Sathishkumar, N.; Toley, B. J. Experimental Measurement of Parameters Governing Flow Rates and Partial Saturation in Paper-Based Microfluidic Devices. Langmuir 2018, 34 (30), 8758-8766.

(20) Kauffman, P.; Fu, E.; Lutz, B.; Yager, P. Visualization and Measurement of Flow in TwoDimensional Paper Networks. Lab Chip 2010, 10 (19), 2614-2617.

(21) Hertaeg, M. J.; Tabor, R. F.; Berry, J. D.; Garnier, G. Journal of Colloid and Interface Science Dynamics of Stain Growth from Sessile Droplets on Paper. J. Colloid Interface Sci. 2019, 541, 312-321. 


\section{Modelling-Guided Design of Paper Microfluidic Networks - A Case Study of Sequential Fluid Delivery}

Dharitri Rath $^{1 \dagger}$, Bhushan J. Toley ${ }^{1 *}$

${ }^{1}$ Department of Chemical Engineering

Indian Institute of Science

Bengaluru, Karnataka 560012

India

Keywords: Paper-based microfluidics, sequential delivery, two-dimensional paper networks multi-step assays, Richard's equation, partial saturation.

* Correspondence to:

Bhushan J. Toley

Department of Chemical Engineering

Indian Institute of Science

C V Raman Avenue

Bengaluru, Karnataka 560012

Phone: +91-80-2293-3114

E-mail: bhushan@iisc.ac.in

\footnotetext{
${ }^{\dagger}$ Current address of Dharitri Rath is: Department of Chemical Engineering Indian Institute of Technology Jammu Jagti, NH-44, PO Nagrota Jammu - 181221 J\&K, India
} 


\section{Supporting Information}

\section{S1. Diffusivity Measurements}

Red, yellow, and orange food coloring dyes were acquired locally and their diffusivity in paper was measured using the following method. Rectangular strips, $0.8 \mathrm{~cm} \times 11 \mathrm{~cm}$, of nitrocellulose NC FF120HP were laminated with PSA on one side. An acrylic humidity chamber was built in-house to maintain a relative humidity of $80 \%$ or above by introducing wet paper towels in the chamber, as in our previous work ${ }^{1}$. These paper devices were first saturated by introducing water into a source well connected to one end and then kept inside the humidity chamber for 30 min to ensure complete saturation. A 1:10 dilution of the stock dye solution was then added into the source well from which dye molecules would diffuse into the paper. Time lapse images were acquired every 10s using a webcam (Logitech C525). Spatiotemporal profiles of dye diffusion were obtained from these images using ImageJ software. Color channels were first split and data from green, blue, and green channels was used to analyze data from red, yellow, and orange dyes, respectively. The movement of dyes in the NC strips can be modelled by a convective diffusive transport equation, given as follows:

$\frac{\partial C_{i}}{\partial t}=D_{e f f} \nabla^{2} C_{i}-v \cdot \nabla C_{i}$

where $C_{i}$ is the concentration of the dye, $D_{\text {eff }}$ is the effective diffusivity of the dye, and $v$ is the convective velocity of the dye molecules. The above equation was solved using "transport of dilute species interface" in COMSOL Multiphysics v5.4. A Dirichlet boundary condition, $C_{i n}$ $=1 \mathrm{~mol} / \mathrm{m}^{3}$, was imposed at the inlet and an outflow boundary condition $\left(\vec{n} \cdot D_{\text {eff }} \nabla C_{i}=0\right)$ was imposed at the other end. Results of the simulation data were matched with the experimental

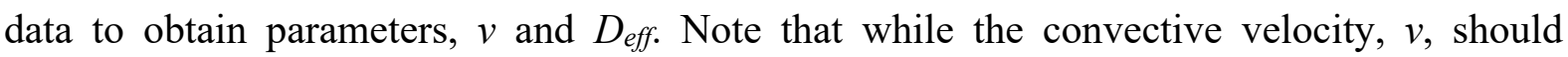
theoretically be zero in the experimental setup, experimental profiles were indicative of a very small convective velocity, likely because of a baseline rate of evaporation. 
Experimental and simulated spatiotemporal concentration profiles of red, yellow, and orange dyes introduced into reservoirs connected to water-soaked NCFF120 strips are shown in Figure S1. Diffusion coefficients for red, yellow, and orange dyes were found to be $1.667 \pm 0.76 \times 10^{-}$ ${ }^{10} \mathrm{~m}^{2} / \mathrm{s}, 3 \pm 0.5 \times 10^{-10} \mathrm{~m}^{2} / \mathrm{s}$, and $1.667 \pm 0.76 \times 10^{-10} \mathrm{~m}^{2} / \mathrm{s}$, respectively $(\mathrm{N}=3)$. Negligible, but non-zero convective velocities of magnitude less than $5 \times 10^{-5} \mathrm{~m} / \mathrm{s}$ were obtained for all three dyes, presumable due to a baseline rate of evaporation.

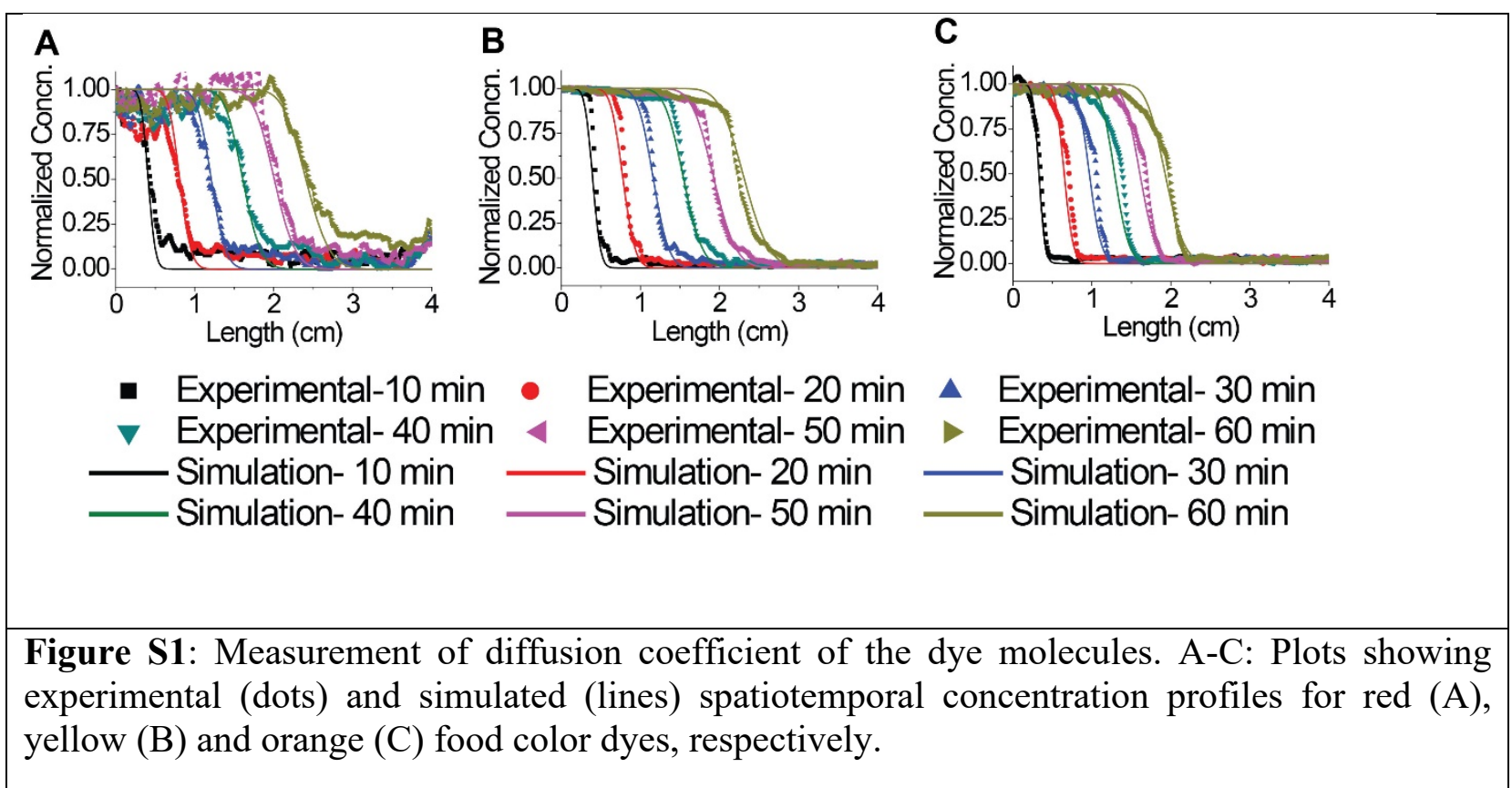

\section{S2. Capillary pressure and permeability versus saturation data}

Plots showing experimental data for the variation of capillary pressure and permeability with saturation for three wicking pad materials are presented in Figure S2. These data were used to obtain the Van Genuchten parameters reported below. The fits were obtained using MATLAB curve fitting tool using the nonlinear regression analysis where $\mathrm{R}^{2} \sim 0.99$. Table $\mathrm{S} 1$ also reports the values of porosities and permeability obtained for the three materials using methods described in our previous paper ${ }^{1}$. 
Table S1: Van Genuchten Parameters

\begin{tabular}{|c|c|c|c|}
\hline Parameter & CFSP & CF3 & CF4 \\
\hline$\alpha$ & 1.4 & 1.1 & 1.4 \\
\hline $\mathrm{n}$ & 1.6 & 1.55 & 1.6 \\
\hline 1 & 0.5 & 0.5 & 0.5 \\
\hline$\theta_{s}$ & 0.9 & 0.85 & 0.85 \\
\hline$\kappa_{S}$ & $4.3 \times 10^{-12}\left[\mathrm{~m}^{2}\right]$ & $8.9 \times 10^{-13}\left[\mathrm{~m}^{2}\right]$ & $2.7 \times 10^{-12}\left[\mathrm{~m}^{2}\right]$ \\
\hline
\end{tabular}

For obtaining partial saturation experimentally, the dimensions of the paper membrane used were $10 \mathrm{~cm} \mathrm{x} 0.8 \mathrm{~cm}$ for CFSP, and $5 \mathrm{~cm} \mathrm{x} 0.8 \mathrm{~cm}$ for CF3 and CF4.

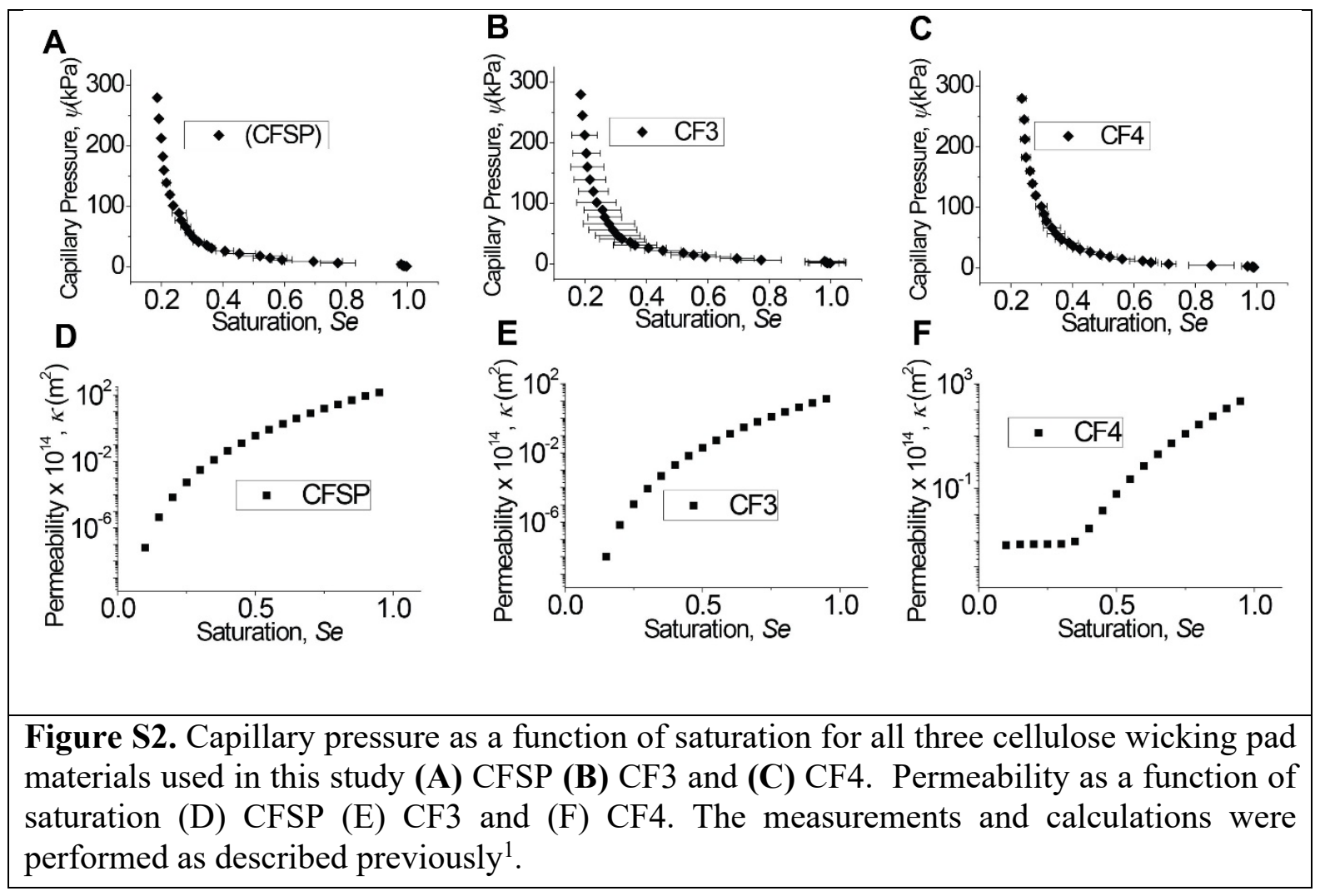

S3. Electrochemical Band marking set-up for the measurement of fluid flow velocity in the test and wicking pad membrane assembly 
For the electrochemical band marking set-up, a $1 \mathrm{mg} / \mathrm{ml}$ solution of phenol red in phosphate buffer saline ( $\mathrm{pH}$ 7.4) was used as the flow fluid. A simple electrochemical circuit powered by a $9 \mathrm{~V}$ battery was set up such that the positive electrode was immersed in the source well and the negative electrode was put in contact with the test membrane, just downstream of the source well. When DC current was passed through the circuit, $\mathrm{OH}^{-}$ions generated by electrolysis of water at the negative electrode increased the $\mathrm{pH}\left(2 \mathrm{H}_{2} \mathrm{O}+2 \mathrm{e}^{-} \leftrightharpoons \mathrm{H}_{2}+2 \mathrm{OH}^{-}\right)$. Because of this shift in $\mathrm{pH}$ to beyond its $p K_{a}$ value (8.0), phenol red that is yellow at $\mathrm{pH} 7.4$ turned deep red (Figure S3). Pulses (3s-long) of DC voltage were generated every 30s for a total duration of 15 min (starting 90s after the fluid front crossed the marking electrode) to generate a series of red bands. The distance traveled by each band in the first minute of its journey was measured using ImageJ to calculate flow velocity.

\section{A}

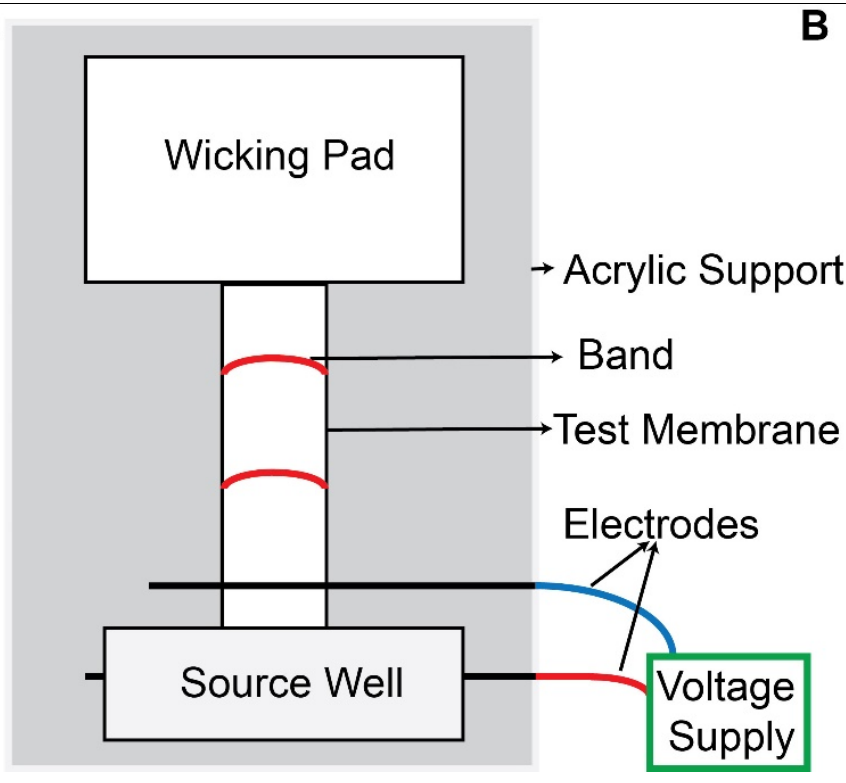

B

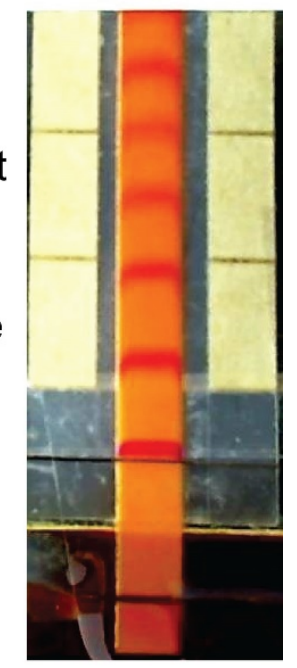

Figure S3. Band-marking set-up developed in-house for the measurement of advancing fluid front with respect to time. Panel (A) shows the paper device being connected to a breadboard and a $9 \mathrm{~V}$ battery, (B) the zoomed in view of the paper device, consisting of a main channels where NC FF120 is used as the test membrane which is attached to a wicking pad made of CFSP cellulose pad.

S4. Derivation of the species transport equation in porous media (equation 4) using differential balance 
The general mass balance equation depicts:

Rate of accumulation $=$ Input + Generation - Output - Consumption S2

For a system with no reactions, mass is neither generated nor consumed, the above equation simplifies to

Rate of accumulation $=$ Input - Output

Figure $\mathrm{S} 4$ shows a differential volume element: $\Delta V=\Delta x \Delta y \Delta z$; Mass of any species in the differential volume: $\Delta m=C_{i}(\Delta x \Delta y \Delta z)$. Since the differential volume is porous, we have to multiply the above equation with $\theta$ in order to obtain the actual mass contained in the differential porous element. Hence, the rate of accumulation of mass in the differential element: $\frac{\partial}{\partial t}\left(\theta C_{i} \Delta x \Delta y \Delta z\right)$. Input (because of diffusive and convective transport): $\left(-\left.D_{\text {eff }, i} \frac{\partial C_{i}}{\partial x}\right|_{x}+\left.v C_{i}\right|_{x}\right) \Delta y \Delta z ;$ Output: $\left(-\left.D_{e f f, i} \frac{\partial C_{i}}{\partial x}\right|_{x+\Delta x}+\left.v C_{i}\right|_{x+\Delta x}\right) \Delta y \Delta z$.

Similarly, we can write the in and out terms contributed by diffusion and convection into the differential volume element. Putting these terms in equation S3, and applying Taylor series expansion we can obtain the final form of the equation as:

$\frac{\partial\left(\theta C_{i}\right)}{\partial t}=\nabla \cdot\left(D_{e f f, i} \nabla C_{i}\right)-v \cdot \nabla C_{i}$

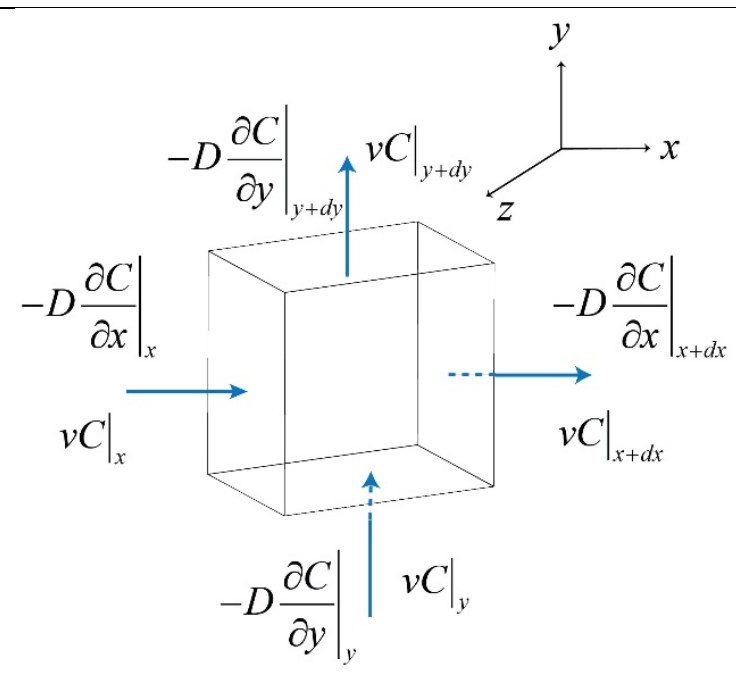


Figure S4: Differential mass balance over an infinitesimal control volume. Rate of input of output of masses resulting from diffusion and convection are presentation in the figure in $\mathrm{x}$ direction.

In order to obtain the spatiotemporal variation of concentration, the above species transport equation, in 'Transport of diluted species in porous media interface' was coupled to 'Richards equation interface' in COMSOL. Table S2 depicts the parameters needed to simulate the coupled interfaces.

Table S2: Parameters used in COMSOL for solving 'Richards equation interface' coupled to 'Transport of diluted species in porous media interface'

\begin{tabular}{ccc} 
Variables & Value & Description \\
\hline$\rho$ & $1000\left[\mathrm{~kg} / \mathrm{m}^{3}\right]$ & Density of fluid \\
$\theta_{s}$ & varied (Table S1) & Porosity of the membrane \\
$\theta_{r}$ & 0 & Residual saturation
\end{tabular}




$\begin{array}{ccc}\kappa_{s} & \text { varied (Table S1) } & \text { Permeability at } 100 \% \\ S & 0 & \text { saturation } \\ \alpha & \text { Varied (Table S1) } & \text { Van Genutchen parameter } \\ n & \text { Varied (Table S1) } & \text { Van Genutchen parameter } \\ l & \text { Varied (Table S1) } & \text { Van Genutchen parameter } \\ \text { D } & \text { Varied (Section S1) } & \text { Diffusivity of the species }\end{array}$

S5. Absorbance measurement, and calibration curve for concentration vs. absorbance for the red color food-dye:

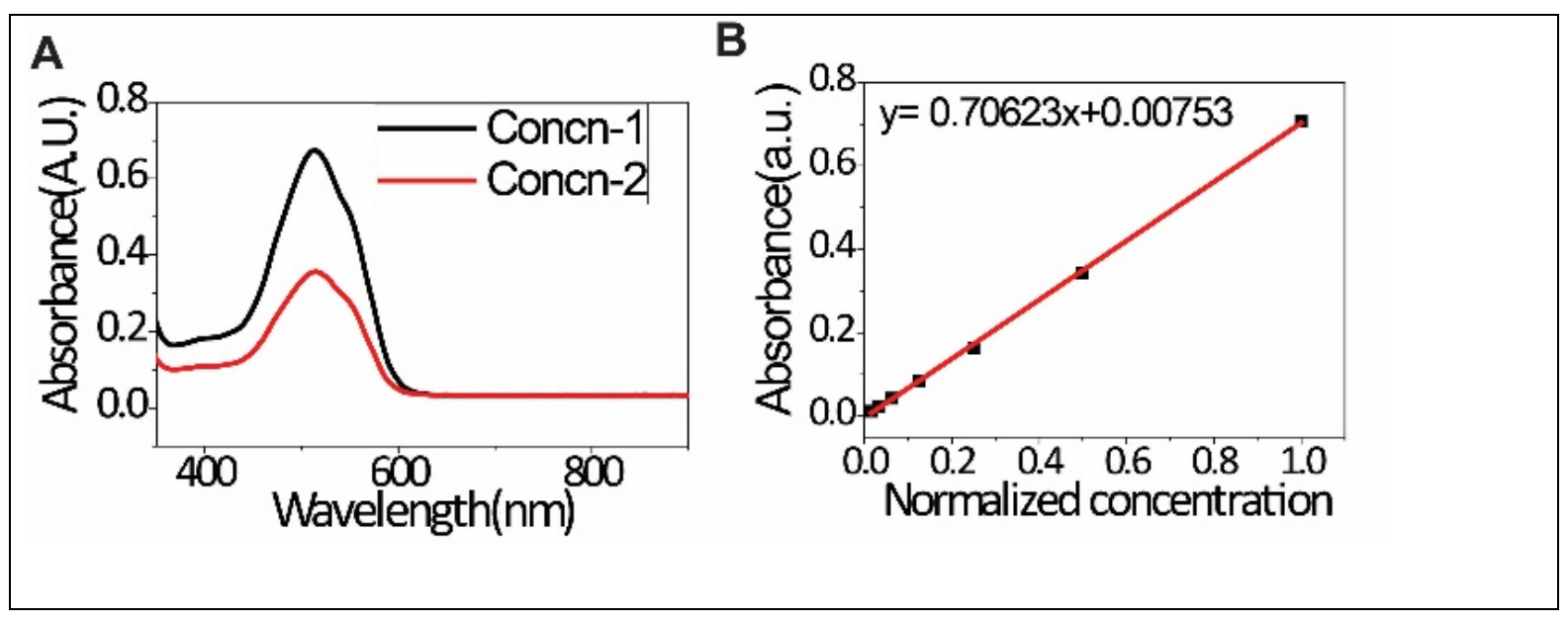


Figure S5: Absorbance measurements of red color food-dye. (A) The absorbance peak ( $520 \mathrm{~nm}$ ) for red dye; (B) Calibration curve obtained at $520 \mathrm{~nm}$ for the known concentrations of the dye.

\section{S6. Protocol used to measure spatiotemporal variation of the concentration of food color} dye inside the paper membrane

The protocol used to measure concentration of dyes being flowed in the rectangular strip is described schematically below.

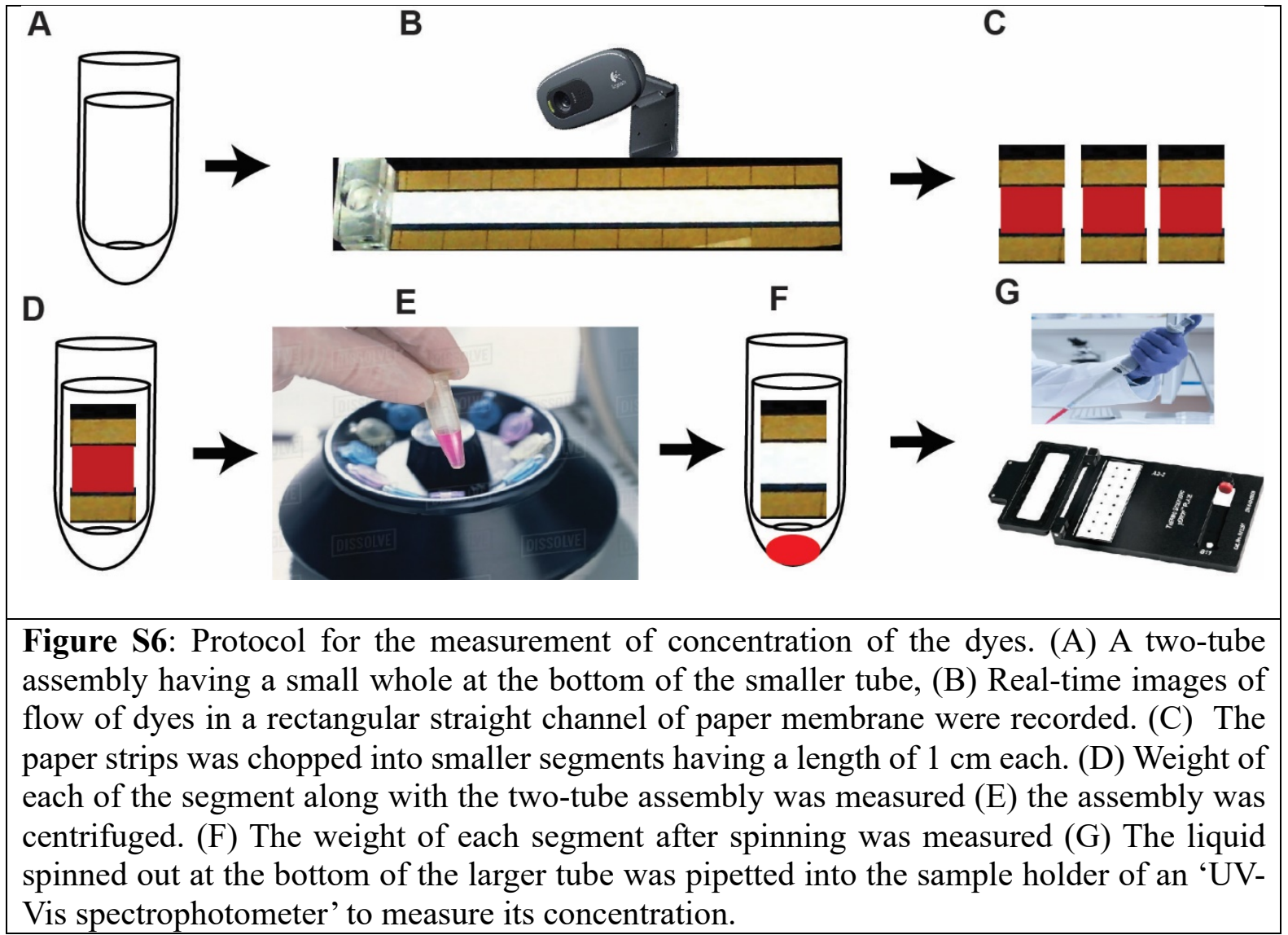

\section{S7. Description of the gold-NP based immunoassay}

A standard sandwich immunoassay similar to the one reported by Toley et.al. ${ }^{2}$ was performed for the detection of malarial antigen PfHRP2. $0.5 \mathrm{mg} / \mathrm{ml}$ of anti- Plasmodium falciparum HRP2 antibody (Mouse IgM) was first hand-spotted at the detection region on the 2DPN. $500 \mathrm{ng} / \mathrm{ml}$ of Plasmodium falciparum HRP2 protein was prepared in a solution of phosphate buffer saline 
containing $1 \%$ BSA with $0.05 \%$ Tween 20 . A second murine antibody to PfHRP2 was conjugated to a gold nanoparticle served as a label. Plasmodium falciparum HRP2 protein and the antibodies were purchased from Immunology Consultants Laboratory (ICL) (Portland, Oregon, USA). The gold conjugation was performed using InnovaCoat ${ }^{\circledR}$ GOLD (20 OD) Conjugation Kit, wherein $0.1 \mathrm{mg} / \mathrm{mL}$ monoclonal PfHRP2 antibodies were mixed with the gold nanoparticles of an initial optical density (OD) of 20. This was further diluted to an OD of 2.0 in conjugate buffer, consisting of $2 \mathrm{mM}$ borate buffer (Thermo Scientific, Waltham, MA) and $10 \%$ (w/v) sucrose (Sigma-Aldrich, St. Louis, MO). Phosphate buffer saline with $0.05 \%$ Tween 20 was used as the wash buffer. A commercially available gold enhancement reagent (Nanoprobes, Yaphank, NY) was used as the signal amplification reagent. Four different solutions (Solutions A, B, C, and D), provided in the kit, were mixed with the prescribed amount and order. Initially, equal volumes of solution A (enhancer) \& B (activator) were mixed, and the solution was kept for 5 minutes. This was followed by the addition of solution C (initiator) \& D (buffer) and mixed thoroughly.

\section{S8: Calculation of Peclet Number}

For this calculation, the characteristic length scale was assumed to be the width of the paper strips used, not the pore diameter. This is because we are concerned with finding whether diffusion or convection dominates transport over the length scale of the device, not within a pore. The values used for calculation were:

Velocity, $v=10^{-4} \mathrm{~m} / \mathrm{s}$ (refer to Fig. 1 in main text)

Length scale, $L=0.5 \mathrm{~cm}$ (refer to section "Modelling sequential delivery in 2DPNs" in main text) 
Diffusivity, $D=10^{-10} \mathrm{~m}^{2} / \mathrm{s}$ (refer to section $\mathrm{S} 1$ )

Therefore,

$P e=\frac{v L}{D}=5000$

\section{References}

(1) Rath, D.; Sathishkumar, N.; Toley, B. J. Experimental Measurement of Parameters Governing Flow Rates and Partial Saturation in Paper-Based Microfluidic Devices. Langmuir 2018, 34, 8758-8766. https://doi.org/10.1021/acs.langmuir.8b01345.

(2) Toley, B. J.; Wang, J. A.; Gupta, M.; Buser, J. R.; Lafleur, L. K.; Lutz, B. R.; Fu, E.; Yager, P. A Versatile Valving Toolkit for Automating Fluidic Operations in Paper Microfluidic Devices. Lab a Chip - Miniaturisation Chem. Biol. 2015, 15 (6), 14321444. https://doi.org/10.1039/c4lc01155d. 